\title{
An Approach to Star Map Simulation for Star Sensor Considering the Effect of Image Motion
}

\author{
Ai-Jun Li, Chao-Shan Liu, Xiao-fang Shen \\ Department of Physics, College of Science, High Technology Research Institute, Xi' an, China \\ Email: Liaijun1979@163.com, Liuchsxian@yahoo.com.cn
}

Received 2013

\begin{abstract}
To test high resolution and dynamic performance of star sensor, a method of consideration image motion on Modeling the motion blur of star sensor is proposed. Firstly, image motion geometric model based on the rotation of Starlight vector is studied. Secondly, with the help of the normal distribution of static star image energy model, introducing the star image motion speed, obtaining the energy distribution function of moving stars, implementing high dynamic simulation of star map. Finally, establishing the simulation environment, through adjusting input parameters such as integral time, rate of change of three attitude angle, the launch time, location, then, important simulation data of stars observed by star sensor in orbit can quickly be obtained, such as navigation stars information, value and direction of image motion, intensity distribution, signal to noise ratio. This work is very important to research and evaluate the star image motion compensation algorithm.
\end{abstract}

Keywords: Star Sensor; Image Motion Model; Simulation of Star Map

\section{Introduction}

In the highly dynamic environment, the relative motion of the star sensor will inevitably cause star image blurred during exposure, so simulation model on star sensor imaging in-orbit must consider the impact of the blurring motion, in order to achieve a real sense of the dynamic simulation of star map.[1] In addition, the movement form of star sensor in-orbit is complex, and blurred star image differently along with projectile motion form varies, but with the aid of simulation software, blur simulation star map contained the single or multi-parameter can be easily obtained, providing the reference for the blur model and simulation of high resolution star sensor, which is very important to research and evaluate the star image motion compensation algorithm. [2-6]

This article studies the mechanism of star sensor motion-blurred image. First of all, it researches on the energy distribution characteristics of movement star image. In the static state, star image energy is the normal distribution in the dynamic case, because the centre of stars image moves continuously during the exposure, limited star light energy is spread in more pixels, and the distribution areas are closely associated with the movement. Second, image motion model based on rotation of star light vector is studied, providing the beneficial reference for compensation of star sensor in-orbit.

\section{The Energy Distribution Characteristics of Moving Stars}

In the static state, star image energy satisfies the normal distribution. In order to obtain a higher star positioning accuracy, star sensor generally uses defocus technique, making stellar image point of the image plane a fuzzy spot that is distributed in the $3 \times 3$ pixels. The energy distribution in the image plane can be expressed by the 2D Gauss function.[7]

$$
I(x, z)=\frac{I_{0}}{2 \pi \sigma_{P S F}^{2}} \exp \left[-\frac{\left(x-x_{c}\right)^{2}+\left(z-z_{c}\right)^{2}}{2 \sigma_{P S F}^{2}}\right]
$$

In the equation: $I_{0}$ refers to the optical signal energy projected onto the photosensitive surface of APS during the $\mathrm{T}$ interval, which is determined by the magnitude, the lens diameter, and the detector quantum efficiency factors. $\left(x_{c}, z_{c}\right)$ is star energy center (centroid), that is the star projected position in the imaging surface of the star sensor. $\sigma_{\mathrm{psf}}$ is Gaussian radius of star point energy distribution, which mainly depends on aberration optimization and image plane defocused position during optical design stage.

In the dynamic state, the star image center has been moving during the star imaging process, namely image motion. Limited star light energy spread in more pixels, and distributed region and movement form is closely related. Usually star sensor's exposure time is short $(<$ 
$300 \mathrm{~ms}$ ), the star point on the image plane can be approximately considered in uniform motion. Suppose the moving velocity of star image in the detector image plane is $\left(v_{x}, v_{z}\right)$, when the exposure time is $\Delta t$, the signal energy of star image that the $(i, j)$ pixel receives is:

$$
\begin{aligned}
I(i, j)=\frac{I_{0}}{2 \pi \sigma_{P S F}^{2}} \int_{0}^{\Delta t} \int_{i-\frac{1}{2}}^{i+\frac{1}{2}} \int_{j-\frac{1}{2}}^{j+\frac{1}{2}} \exp [ & -\frac{\left(x-x_{c}-v_{x} t\right)^{2}}{2 \sigma_{P S F}^{2}} \\
+ & \left.\frac{\left(z-z_{c}-v_{z} t\right)^{2}}{2 \sigma_{P S F}^{2}}\right] d x d z d t
\end{aligned}
$$

The $(i, j)$ value is to take $\left(x_{c}, z_{c}\right)$ as the central neighborhood, depending on star image distribution area.

\section{The Image Motion Model of Star Sensor}

Carrier movement generally is a compound movement combined by the linear motion, angular motion, vibration combination, and image motion of star block along with carrier varies. The influence of carrier angular motion on image motion and energy distribution of stars is the largest.

Suppose the angle between star light direction and optical axis direction $\left(y_{s}\right)$ is $\alpha$ at $t$ time, as shown in Figure 1. Star image on point $p$ of image plane. Within the exposure time, vector of Starlight turn of angle $\Delta \alpha=$ $\omega_{S} \cdot \Delta t$, and the light spot in the image plane moves from point $p$ to point $p^{\prime}$. Takes $o_{A P S}$ as the center of circle, $o_{A P S} p$ as the radius of arc, $o_{A P S} p$ and $o_{A P S} p^{\prime}$ cross at $\mathrm{p}^{\prime \prime}$ point, the turning angle is $\Delta \alpha^{\prime}=\omega_{S y} \cdot \Delta t$. Image motion $L_{p p^{\prime}}$ can be viewed as synthesis of tangential image motion $L_{p p^{\prime \prime}}$ and star image $o_{A P S} p^{\prime}$ along radial direction $L_{p^{\prime \prime} p^{\prime}}$ from selecting the $p^{\prime \prime}$ movement to $p^{\prime}$ points. And $L_{p^{\prime \prime} p^{\prime}}$ may be obtained by the star light vector $z_{A P S}$-rotation and $x_{A P S}$-rotation respectively rotate $\Delta \alpha^{\prime \prime}=\omega_{S z} \cdot \Delta t, \quad \Delta \alpha^{\prime \prime \prime}=\omega_{S x} \cdot \Delta t$. According to the geometric relations, it is easy to obtain star image motion in $z_{A P S}$ and $x_{A P S}$ axial direction quantity respectively is: $[9,10]$

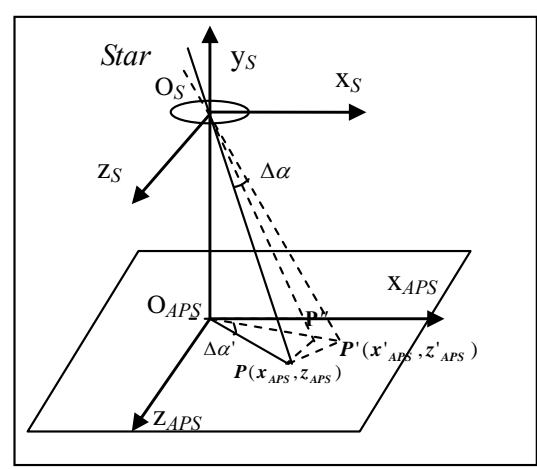

Figure 1. Geometric relationship between the star sensor image motion.

$$
\begin{aligned}
& L_{\left(p^{\prime \prime} p^{\prime}\right) z}=f \cdot\left[\tan \left(\alpha+\Delta \alpha^{\prime \prime}\right)-\tan (\alpha)\right] \\
& L_{\left(p^{\prime \prime} p^{\prime}\right) x}=f \cdot\left[\tan \left(\alpha+\Delta \alpha^{\prime \prime}\right)-\tan (\alpha)\right]
\end{aligned}
$$

Because $\Delta \alpha^{\prime}$ is small, $\Delta p p^{\prime} p^{\prime \prime}$ can be treated as right triangle, then:

$$
L_{p p^{\prime}}=\sqrt{L_{p p^{\prime \prime}}^{2}+L_{\left(p^{\prime \prime} p^{\prime}\right) x}^{2}+L_{\left(p^{\prime \prime} p^{\prime}\right) z}^{2}}
$$

In which,

$$
L_{p p^{\prime \prime}}=f \cdot \tan (\alpha) \cdot \Delta \alpha^{\prime}
$$

Carries on Taylor series expansion to (3), and takes its linear function, then:

$$
\begin{aligned}
\tan \left(\alpha+\Delta \alpha^{\prime \prime}\right) & =\tan (\alpha)+\left(1+\tan ^{2}(\alpha)\right) \Delta \alpha^{\prime \prime} \\
L_{\left(p^{\prime \prime} p^{\prime}\right) z} & =f \cdot\left(1+\tan ^{2}(\alpha)\right) \Delta \alpha^{\prime \prime}
\end{aligned}
$$

If the field of view $<10^{\circ}, \tan ^{2}(\alpha) \approx 0$, (7) can be further simplified to:

$$
L_{\left(p^{\prime \prime} p^{\prime}\right) z}=f \cdot \omega_{S z} \cdot \Delta t
$$

Similarly:

$$
L_{\left(p^{\prime \prime} p^{\prime}\right) x}=f \cdot \omega_{S x} \cdot \Delta t
$$

Finally the image motion expression is:

$$
\begin{aligned}
L_{p p^{\prime}} & =f \cdot \Delta t \cdot \sqrt{\omega_{S x}^{2}+\omega_{S z}^{2}+\omega_{S y}^{2} \cdot \tan ^{2}(\alpha)} \\
& \approx f \cdot \Delta t \cdot \sqrt{\omega_{S x}^{2}+\omega_{S z}^{2}}
\end{aligned}
$$

The image moving speed can also be expressed as tangential and radial velocity:

$$
\begin{aligned}
v & =f \cdot \tan (\alpha) \cdot \omega_{S y} \boldsymbol{\tau}+f \cdot \sqrt{\omega_{S x}^{2}+\omega_{S z}^{2}} \mathbf{n} \\
& =v_{\tau}+v_{n}
\end{aligned}
$$

The $\omega_{S}$ component can be obtained according to transformation relations between coordinate systems:

$$
\left[\omega_{S x}, \omega_{S y}, \omega_{S z}\right]^{\mathrm{T}}=\mathbf{C}_{b S}^{\mathrm{T}} \cdot \mathbf{C}_{I b}^{\mathrm{T}}\left[\omega_{I x}, \omega_{I y}, \omega_{I z}\right]^{\mathrm{T}}
$$

$\omega_{I}$ is the angular velocity relative to the inertial system surveyed by SINS, without error accumulation, but $\omega_{S}$ has component error because of the error accumulation of $C_{I b}$ led.

The star image intensity distribution may also be expressed under polar coordinate system:

$$
I(\theta, r)=\frac{I_{0}}{2 \pi \sigma_{P S F}^{2}} \exp \left[-\frac{\left(\theta-\theta_{c}\right)^{2}+\left(r-r_{c}\right)^{2}}{2 \sigma_{P S F}^{2}}\right]
$$

\section{Numerical Analysis of Image Shift}

\subsection{Imagemotion Simulation Caused by the Change in Attitude Variation}

Carrier attitude angle $\left(\phi_{0}, \psi_{0}, \gamma_{0}\right)$ is $\left(20^{\circ}, 3^{\prime \prime} / 60,3^{\prime \prime}\right.$ /600) when viewing the star. Its rate of change $\dot{\phi}, \dot{\psi}, \dot{\gamma}$ 
is $(0.3,0.05,0.05)^{\circ} / \mathrm{s}$. The exposure time is $0.3 \mathrm{~s}$. Star sensor's main focus $f=52 \mathrm{~mm}$. $\omega_{I}$ can be computed according to (13)

$$
\left[\omega_{I x}, \omega_{I y}, \omega_{I z}\right]^{\mathrm{T}}=\left[\begin{array}{ccc}
-\sin \psi & 0 & 1 \\
-\cos \psi \sin \gamma & \cos \gamma & 0 \\
\cos \psi \cos \gamma & -\sin \gamma & 0
\end{array}\right][\dot{\phi}, \dot{\psi}, \dot{\gamma}]^{\mathrm{T}}
$$

Tracing a guide star, its serial number, the magnitude, the right ascension, the declination respectively is 637, 3.0 Mv, 2.9227 rad, 0.7764 rad. The position of reference star in the image plane can be obtained at 6 continual time points, as shown in Table $\mathbf{1}$.

According to the (9) and (10), the image moving velocity value and image motion can be obtained, and pixel size is $0.025 \mathrm{~mm}$, see Table 2:

\subsection{Moving Star Image Simulation}

According to the need of star image subdivision algorithm, when star energy distribution window size takes the $3 \times 3$ element, the star energy in the $2 \times 2$ element gathering cannot be lower than $80 \%$ (degree of energy concentration $\left.K=I_{\text {center }} / I_{\text {facula }}\right)$. For example, when taking $\sigma_{p s f}=0.55$, The energy of star signal in the $3 \times 3$ element and $2 \times 2$ element is:

$$
\begin{aligned}
& \mathrm{I}_{3 \times 3}=\int_{-1.5}^{1.5} \int_{-1.5}^{1.5} I(x, z) d x d z=0.987 \\
& \mathrm{I}_{2 \times 2}=\int_{-1}^{1} \int_{-1}^{1} I(x, z) d x d z=0.867
\end{aligned}
$$

At this time the energy of central element was 0.405; the degree of energy concentration was $40.5 \%$; the energy distribution of Gaussian distribution simulation of Star map can be obtained. Energy within the window size and energy concentration of k-value is only related with parameter $\sigma_{\mathrm{PSF}}$ in Gaussian distribution function.

In star sensor imaging process, if the center of star moves, after the migration, Asterism central position can be indicated with $\left(x_{c}+v_{x} \cdot \Delta t / 2, z_{c}+v_{z} \cdot \Delta t / 2\right)$. In this simulation condition, the center gray of the dynamic is only $43.3 \%$ of that in static state, and stars image grey-scale distribution (size and strength) has also changed, as shown in Figure 2. The motion blur star map considered image motion, as shown in Figure 3.

\section{Conclusions}

Image motion model of star sensor obtained according to revolving of star light vector is very direct-viewing, and it can quite real simulation celestial chart that collected

Table 1. Six Coordinates on the Plane of Guide Star(mm).

\begin{tabular}{lllllll}
\hline $\boldsymbol{x}$ & -2.406 & -2.472 & -2.539 & -2.605 & -2.671 & -2.738 \\
\hline $\boldsymbol{Z}$ & 1.201 & 1.217 & 1.233 & 1.249 & 1.265 & 1.281 \\
\hline
\end{tabular}

Table 2. Image Moving Velocity And Image Drift (Pixel Integer).

\begin{tabular}{cccccc}
\hline $\boldsymbol{V} \boldsymbol{x}$ & -8.72 & -8.72 & -8.72 & -8.72 & -8.72 \\
\hline $\boldsymbol{V z}$ & 1.96 & 1.96 & 1.96 & 1.96 & 2.0 \\
$\boldsymbol{L}$ & 2.726 & 2.726 & 2.726 & 2.726 & 2.726 \\
\hline
\end{tabular}

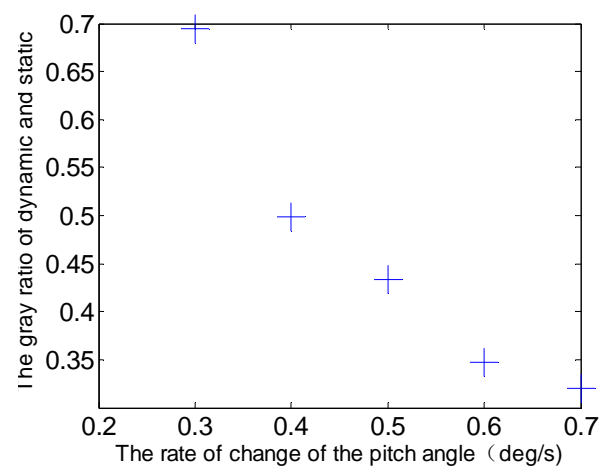

Figure 2. The gray ratio of dynamic and static.

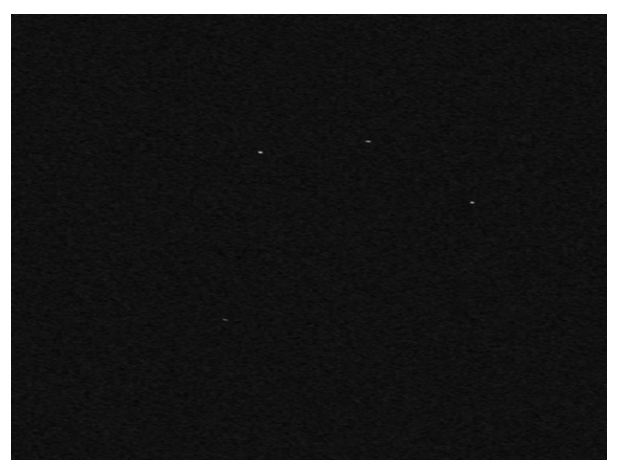

Figure 3. Star map of motion blur considered the image motion.

by the star sensor in-orbit rate process. By adjusting the input parameters, such as the integration time, three posture angles and its changing rate, then the guide star information, image motion, orientation, grayscale distribution, such as signal-to-noise ratio, can easily be obtained.

\section{REFERENCES}

[1] M. A. Samaan “Toward Faster and More Accurate Star Sensors Using Recursive Centroiding and Star Identification,” Texas A\&M University, 2003.

[2] Y. Jin, J. Jiang and G. J. Zhang, "Star Extraction Method for High Dynamic Star Sensor,” Infrared and Laser Engineering, Vol. 40, No.11, 2011, pp. 2281-2285.

[3] B. L. Jiao and X. H. Yan, "Image-Motion Analysis and Image Restoration Based on tdi-ccd Imaging," Journal of Astronautics, Vol. 29, No. 2, 2008, pp. 675-678.

[4] X. Li and H. Zhao, "Analysis of Star Image Centroid Accuracy of an APS Star Sensor in Rotation,"Aerospace Control and Application, Vol. 35, No. 4, 2009, pp.11-16. 
[5] W. Quan and W. N. Zhang, "Restoration of Motion-Blurred Star Image Based on Wiener Filter," 4th International Conference on Intelligent Computation Technology and Automation, Vol. 2, 2011, pp. 691-694.

[6] B. M. Miller and E.Y., "Rubinovich Image Motion Compensation at Charge-Coupled Device Photo-Graphing in Delay-Integration Mode. Automation and Remote Control, 2007, Vol. 68, No. 3, pp. 564-571. doi:10.1134/S0005117907030162

[7] H. B. Liu, D. Z. Su, J. C.Tan, J. K. Yang and X. J. Li, "An Approach to Star Image Simulation for Star Sensor Considering Satellite Orbit Motion and Effect of Image
Shift,” Journal of Astronautics, Vol. 32, No. 05, 2011, pp. 1190-1184.

[8] C. S. Liu, R. P. Ma, C. G. Xiao and G. B. Liu, "Star Pattern Matching for Celestial Guidance,” Journal of Astronautics, Vol. 27, No.1, 2006, pp. 31-35.

[9] X. J. Wu and X. L. Wang, "Motion Blur of Star Image and Restoration," Journal of Beijing University of Aeronautics and Astronautics, 2011, Vol. 37, No. 11,pp. 1-5.

[10] C. S. Liu, G. B. Liu, X. G. Wang and A. H. Li, "Star Sensor Principle and Application Strapped on Missile,” National Defense Industry Press, Beijing, 2010, p. 205. 